NASA Technical Memorandum 102639

AVSCOM Technical Memorandum 90-B-006

\title{
Differential Equation Based Method For Accurate Approximations in Optimization
}

Jocelyn I. Pritchard and Howard M. Adelman

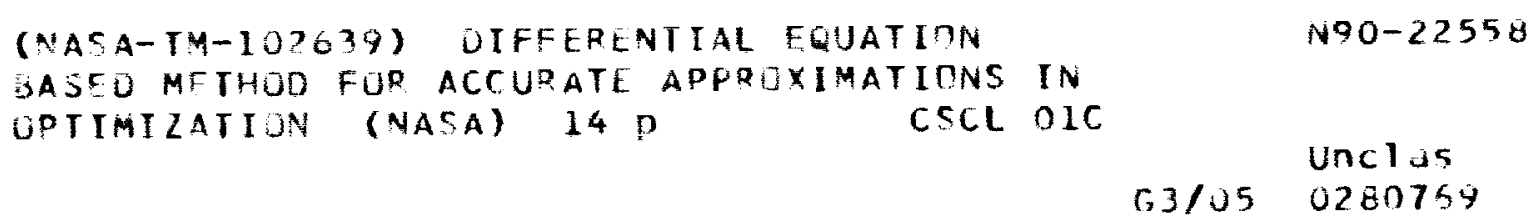

April 1990

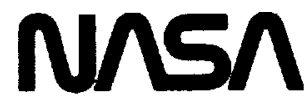

National Aeronautics and

Space Administration

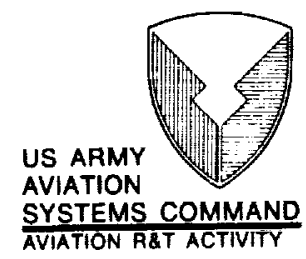




\title{
DIFFERENTIAL EQUATION BASED METHOD FOR ACCURATE APPROXIMATIONS IN OPTIMIZATION
}

\author{
Jocelyn I. Pritchard" \\ U. S. Army Aerostructures Directorate \\ USAARTA-AVSCOM \\ Langley Research Center \\ Hampton, Virginia \\ and \\ Howard M. Adelman** \\ National Aeronautics and Space Administration \\ Langley Research Center \\ Hampton, Virginia
}

\section{Abstract}

This paper describes a method to efficiently and accurately approximate the effect of design changes on structural response. The key to this new method is to interpret sensitivity equations as differential equations that may be solved explicitly for closed form approximations, hence, the method is denoted the Differential Equation Based (DEB) method. Approximations were developed for vibration frequencies, mode shapes and static displacements. The DEB approximation method was applied to a cantilever beam and results compared with the commonly-used linear Taylor series approximations and exact solutions. The test calculations involved perturbing the height, width, cross-sectional area, tip mass, and bending inertia of the beam. The DEB method proved to be very accurate, and in most cases, was more accurate than the linear Taylor series approximation. The method is applicable to simultaneous perturbation of several design variables. Also, the approximations may be used to calculate other system response quantities. For example, the approximations for displacements are used to approximate bending stresses.

\begin{tabular}{ll} 
& \multicolumn{1}{c}{ Nomenclature } \\
$\mathbf{K}$ & stiffness matrix \\
$\mathbf{M}$ & mass matrix \\
$\mathbf{S}$ & number of steps \\
$\mathbf{v}$ & design variable \\
$\mathbf{v}$ & vector of design variables \\
$\Delta v$ & change in design variable
\end{tabular}

- Research Engineer, Interdisciplinary Research Office, Member AHS

- Deputy Head, Interdisciplinary Research Office Member AIAA,ASME

$\omega^{2}$
$\mathbf{\Phi}$
$\theta$
Superscript
$T$
Subscript
0
$\mathbf{i}$

vibration eigenvalue (frequency) vibration eigenvector (mode shape) distance along move direction

designates transpose

designates nominal value

ith component of vector

\section{Introduction}

It is highly desirable in optimization to be able to estimate the effect of design changes on system behavior without having to perform expensive finiteelement analyses inside an optimization loop. This need has led to an increased interest in the development of accurate and efficient approximation techniques. Presently, many optimization procedures use linear Taylor series approximations of the objective function and constraints so that excessive calls to the full analysis can be eliminated. ${ }^{1}$ Taylor series and other approximations ${ }^{2}$ are also used outside of optimization or quick assessment of the effect of design changes. Other techniques and demonstration of their benefits are described in references 3-11. In this paper an efficient method is described which significantly extends the range of applicability of approximations beyond the range of the linear Tayior series approach. The key to this new approach is to recognize that the formulas for the sensitivity derivatives of system response quantities can be interpreted as differential equations which may be solved to obtain closed form approximations. Herein, these approximations will be referred to as the Differential Equation Based approximations.

\section{Basis of DEB Method}

In this section of the paper the DEB method is developed for approximating the effect of design 
changes on response. First, the method will be developed for the approximation of frequencies, then mode shapes, and finally for static displacements.

\section{Aporoximation of Frequencies}

From reference 12, the equation for the derivative of a non-repeated vibration eigenvalue, $\omega^{2}$ with respect
to a design variable, $v$ is

$$
\frac{d \omega^{2}}{d v}=\boldsymbol{\Phi}^{\top}\left[\frac{d \mathbf{K}}{d v}-\omega^{2} \frac{d \mathbf{M}}{d v}\right] \boldsymbol{\Phi}
$$

where $K$ is the stiffness matrix, $M$ is the mass matrix and $\Phi$ is the eigenvector normalized such that $\Phi^{\top} \mathbf{M} \Phi=1$. Define two scalar quantities $a$ and $b$ as follows

$$
a=\Phi^{\top} \frac{d M}{d v} \Phi \text { and } \quad b=\Phi^{\top} \frac{d K}{d v} \Phi
$$

then Equation (1) may be written as

$$
\frac{d \omega^{2}}{d v}=b-a \omega^{2}
$$

Equation (3) may be interpreted as a first order differential equation in $\omega^{2}$ with variable coefficients. If a and $b$ do not vary excessively with $v$, they may be evaluated at the nominal design and considered to be constant. Then the general solution to equation (3), provided $a$ is nonzero is

$$
\omega^{2}=C e^{-a v}+\frac{b}{a}
$$

Solving for $C$ from the condition of $\omega^{2}=\omega_{0}^{2}$ when $v=v_{0}$ and substituting into Equation (4) gives an explicit approximation for $\omega^{2}$ as

$$
\omega^{2}=\left(\omega_{0}^{2}-\frac{b}{a}\right) e^{-a\left(v-v_{0}\right)}+\frac{b}{a}
$$

\section{Aporoximation of Mode Shapes}

From reference 13, the equation for the derivative is the mode shape, $\Phi$ with respect to a design variable

$$
\frac{d \Phi}{d v}=Q+D \Phi
$$

where $Q$ satisfies the equation

$$
\left[K-\omega^{2} \mathbf{M}\right] \mathbf{Q}=\frac{d \omega^{2}}{d v} \mathbf{M} \Phi-\frac{d K}{d v} \Phi+\omega^{2} \frac{d M}{d v} \Phi
$$

and

$$
D=-\Phi^{T} M Q-\frac{1}{2} \Phi^{T} \frac{d M}{d v} \Phi
$$

Derivations of $Q$ and $D$ may be found in reference 13 . Equation (6) is a nonlinear first order vector differential equation in $\Phi$ with variable coefficients. In order to solve this equation, $Q$ and $D$ are evaluated at the nominal design and assumed constant. Equations (1)

and (5) may be used for explicit forms of $\frac{d \omega^{2}}{d v}$ and $\omega^{2}$ in the calculations of $Q$ and $D$. The solution to equation
(6) is

$$
\Phi=A e^{D v}-\frac{1}{D} Q
$$

where $A$ is determined from the nominal condition that $\Phi=\Phi_{0}$ when $v=v_{0}$. Solving for $A$ and substituting into equation (9) gives the explicit approximation for $\Phi$ as

$$
\Phi=\left(\Phi_{0}+\frac{1}{D} Q\right) e^{D\left(v-v_{0}\right)}-\frac{1}{D} Q
$$

Equation (10) is a vector equation, however, it is uncoupled in the sense that each component of $\Phi$ varies independently with the design variable, $v$. The difference between components is reflected in the corresponding components of $\Phi$ and $Q$.

\section{Appreximation of Displacements}

Assuming a linear elastic structure in which the applied force does not vary with $v$, the equation for the displacement derivative is

$$
\frac{d \mathbf{U}}{d \mathbf{V}}=\mathbf{B U}
$$

where

$$
B=-K^{-1} \frac{d K}{d v}
$$

Equation (11) is a differential equation that can be displacement, $U$. Similar approximation of the equations for frequencies and solving the differential 
B is evaluated at the nominal design and assumed constant for moderate excursions from the nominal design. Equation (11) is a vector differential equation having the following solution

$$
U=e^{B V} A
$$

where $e^{B}$ is the matrix exponential defined as

$$
\mathrm{e}^{B} \equiv \mathrm{I}+\mathrm{B}+\frac{\mathrm{B}^{2}}{2 !}+\frac{\mathrm{B}^{3}}{3 !}+\cdots
$$

Solving for $A$ in equation (13) from the condition that $\mathrm{U}=\mathrm{U}_{0}$ at $\mathrm{V}=\mathrm{v}_{0}$ gives

$$
\mathbf{U}=e^{\mathbf{B}\left(v-v_{0}\right)} \mathbf{U}_{0}
$$

Using equation (14) in equation (15) gives the displacement approximation

$$
U=U_{0}+B\left(v-v_{0}\right) U_{0}+\frac{B^{2}\left(v-v_{0}\right)^{2}}{2 !} U_{0}+\cdots
$$

Rather than compute the matrix $B$ and its powers and then multiply by $U_{0}$, the $B^{n} U_{0}$ terms were obtained iteratively as follows

$$
\begin{aligned}
& K B U_{0}=-\frac{d K}{d v} U_{0} \\
& K B^{2} U_{0}=-\frac{d K}{d v} B U_{0} \\
& \vdots \\
& K B^{n} U_{0}=-\frac{d K}{d v} B^{n-1} U_{0}
\end{aligned}
$$

The Taylor series expansion of $U$ is

$$
U=U_{0}+\frac{d U}{d v}\left(v-v_{0}\right)+\frac{1}{2 !} \frac{d^{2} U}{d v^{2}}\left(v-v_{0}\right)^{2}+\cdots
$$

As pointed out in reference 14, when $K$ is a linear function of $v$, equation (18) can be written as

$$
U=U_{0}+B U_{0}\left(v-v_{0}\right)+B^{2} U_{0}\left(v-v_{0}\right)^{2}+\cdots
$$

Also pointed out in reference 14 , equations (16) and (19) differ only by the factorial in each term. Equation (16) is less accurate than the Taylor series equation (19) when they both converge. Equation (16) is based on the power series for the exponential and will converge for any value of $\Delta v$, whereas the Taylor series may not always converge. Since the terms in both equations (16) and (19) are easily evaluated using equation (17). the DEB method would not be the preferred approximation unless the Taylor series did not converge.

\section{DEB Method Extended to Multiple Design Variables}

The approximations in equations 5,10 , and 15 are directly applicable only when a single design variable is perturbed. To calculate the effect of simultaneous changes in several design variables, the DEB method is modified as follows. If $\mathbf{v}_{0}$ is the vector of nominal design variables and $\mathbf{v}$ is the vector of perturbed design variables, then $v-v_{0}$ is the direction of the move from the nominal to the perturbed design. Any design along the move direction may be associated with a scalar $\theta$ between 0 and 1 . Then

$$
\mathbf{v}(\theta)=\mathbf{v}_{\mathbf{0}}+\theta\left(\mathbf{v}-\mathbf{v}_{\mathbf{0}}\right)
$$

In particular, $\mathbf{v}(1)=\mathbf{v}$ and $\mathbf{v}(0)=\mathbf{v}_{\mathbf{0}}$. Now the sensitivity equations (1), (6), and (11) may each be written with $\theta$ as the independent variable. For example, equation (1) is rewritten as

$$
\frac{d \omega^{2}}{d \theta}=\Phi^{\top}\left[\frac{d \mathbf{K}}{d \theta}-\omega^{2} \frac{d \mathbf{M}}{d \theta}\right] \Phi
$$

or

$$
\frac{d \omega^{2}}{d \theta}=b_{\theta}-a_{\theta} \omega^{2}
$$

where

$$
a_{\theta}=\Phi^{\top} \frac{d M}{d \theta} \Phi \quad \text { and } \quad b_{\theta}=\Phi^{\top} \frac{d K}{d \theta} \Phi
$$




$$
\begin{aligned}
& \frac{d \mathbf{M}}{d \theta}=\sum_{i=1}^{n} \frac{d \mathbf{M}}{d v_{i}} \frac{d v_{i}}{d \theta}=\sum_{i=1}^{n} \frac{d \mathbf{M}}{d v_{i}}\left(v_{i}-v_{o i}\right) \\
& \frac{d K}{d \theta}=\sum_{i=1}^{n} \frac{d K}{d v_{i}} \frac{d v_{i}}{d \theta}=\sum_{i=1}^{n} \frac{d K}{d v_{i}}\left(v_{i}-v_{o i}\right)
\end{aligned}
$$

where $n$ is the number of design variables. Now the solution to equation (21b) is

$$
\omega^{2}(\theta)=\left(\omega_{\sigma}^{2}-\frac{b_{\theta}}{a_{\theta}}\right) e^{-a_{\theta}\left(\theta-\theta_{0}\right)}+\frac{b_{\theta}}{a_{\theta}}
$$

Using equation (20) $\omega^{2}(1)=\omega^{2}(v)$ and by definition $\theta_{0}=0$ and $\theta=1$ at the perturbed design. Therefore, the final form of equation (24) is

$$
\omega^{2}(v)=\left(\omega_{0}^{2}-\frac{b_{\theta}}{a_{\theta}}\right) e^{-a_{\theta}}+\frac{b_{\theta}}{a_{\theta}}
$$

Equation (25) corresponds to one step $\left|\mathbf{v}-v_{0}\right|$. For a step size of $s\left|\mathbf{v}-\mathbf{v}_{\mathbf{o}}\right|$

$$
\omega^{2}(v)=\left(\omega_{\theta}^{2}-\frac{b_{\theta}}{a_{\theta}}\right) e^{-a_{\theta} s}+\frac{b_{\theta}}{a_{\theta}}
$$

Extension of the method to multiple design variables has also been carried out for $\Phi$ and $U$.

\section{DEB Method Extended To Nonlinear Desion Variables}

We will define a nonlinear design variable to be one that contributes nonlinearly to either the mass or stiffness matrix and a linear variable is one that contributes linearly to both the mass and stiffness matrices. An early attemot at applying the DEB method in a straight forward manner to nonlinear design variables proved to be highly inaccurate. In retrospect, the reason for the inaccuracy was that the coefficients which were assumed constant (e.g. $a$ and $b$ ) were in fact highly nonlinear functions of the design variable. The solution to this difficulty is as follows. Define $y$ to be a vector of nonlinear design variables and $v$ to be a vector of linear variables. It is assumed that the linear variables may be written as functions of the nonlinear design variable such that $v_{0}=v\left(y_{0}\right)$ and $v=v(y)$. For example, the bending inertia $I$ and the cross-sectional area $A$ contribute linearly to $M$ and $K$ and they are both functions of the nonlinear variable $H$. Then equation (20) may be rewritten with $v$ as a function of $y$ and $\theta$

$$
\mathbf{v}(\theta, \mathbf{y})=\mathbf{v}_{0}+\theta\left(\mathbf{v}-\mathbf{v}_{0}\right)=\mathbf{v}\left(\mathbf{y}_{0}\right)+\theta\left[\mathbf{v}(\mathbf{y})-\mathbf{v}\left(\mathbf{y}_{0}\right)\right]
$$

The method for nonlinear design variables then becomes very similar to the DEB method for multiple linear variables (equation 26). For this case

$$
\begin{aligned}
& \frac{d \mathbf{M}}{d \theta}=\sum_{i=1}^{n} \frac{d \mathbf{M}}{d v_{i}} \frac{d v_{i}}{d \theta}=\sum_{i=1}^{n} \frac{d \mathbf{M}}{d v_{i}}\left[v_{i}(y)-v_{i}\left(y_{0}\right)\right] \\
& \frac{d \mathbf{K}}{d \theta}=\sum_{i=1}^{n} \frac{d \mathbf{K}}{d v_{i}} \frac{d v_{i}}{d \theta}=\sum_{i=1}^{n} \frac{d K}{d v_{i}}\left[v_{i}(y)-v_{i}\left(y_{0}\right)\right]
\end{aligned}
$$

Then $a_{\theta}$ and $b_{\theta}$ are calculated from equation (22). Unlike the method for linear variables, $a_{\theta}$ and $b_{\theta}$ are evaluated at each step along the move direction due to the nonlinearity of the perturbed variable and in equation (26) $s$ is always equal to 1 . Evaluating $a_{\theta}$ and $b_{\theta}$ involves a recalculation of $v_{i}(y)$ for each perturbed step of $y$ and the multiplication of a matrix by a scalar. The derivatives of the mass and stiffness matrices do not have to be recalculated. They are evaluated once at the nominal design and used throughout the range of the move direction. Again, a similar extension has been carried out for $\Phi$ and $U$.

\section{Results}

The approximations for frequencies, mode shapes, and displacements were tested on a 193 inch cantilever beam modeled with ten equal elements isee fig.1). The beam has a box cross-section and a tip mass. Properties of the beam are listed in table 1.

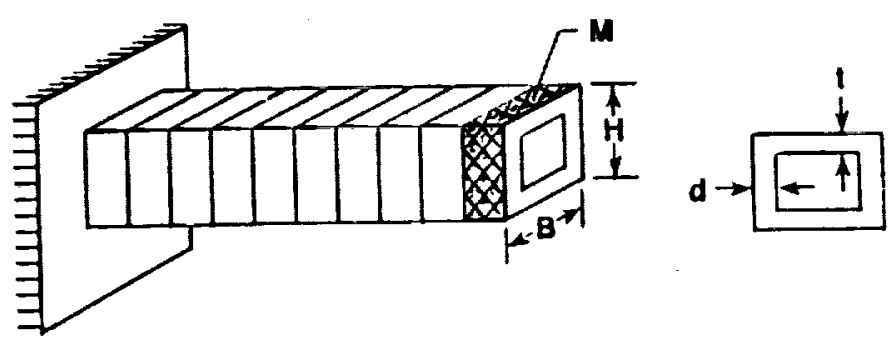

Fig.1 Test problem. 
Table 1 Material properties and cross-sectional dimensions of beam model

\begin{tabular}{|c|c|c|}
\hline Element No. & 1 & $2-10$ \\
\hline$E$ (psi) & $4.90 E 6$ & $5.85 E 6$ \\
\hline$p\left(\right.$ lb/in $\left.^{3}\right)$ & 0.07 & 0.07 \\
\hline$H$ (in.) & 5.00 & 5.00 \\
\hline 8 (in.) & 3.75 & 3.75 \\
\hline$t$ (in.) & 0.80 & 0.80 \\
\hline$d$ (in.) & 0.10 & 0.10 \\
\hline
\end{tabular}

The test cases involved perturing the height $H$, width $B$, cross-sectional area $A$, tip mass $M$, and bending inertia $I$ of the beam. All calculations were generated in a general purpose finite-element program $(E A L)^{15}$. Results were compared with exact solutions and the linear Taylor series approximation.

\section{Erequency Approximation Results}

The DEB method shows excellent capability for approximating frequencies of perturbed designs. For example, figure 2 shows a graph of the fundamental eigenvalue versus the height of the beam, $H$ for values perturbed from the nominal value of 5.0 inches. For as much as a 50 percent increase in $H$, the new approximation is within 2 percent of the exact solution compared to 12 percent for the linear Taylor series approximation. It is also evident from the figure that beyond a 45 percent decrease in $\mathrm{H}$, the linear Taylor series method gives negative values for the eigenvalues. This is an example of the DEB method applied to a nonlinear variable. Figure 3 shows a

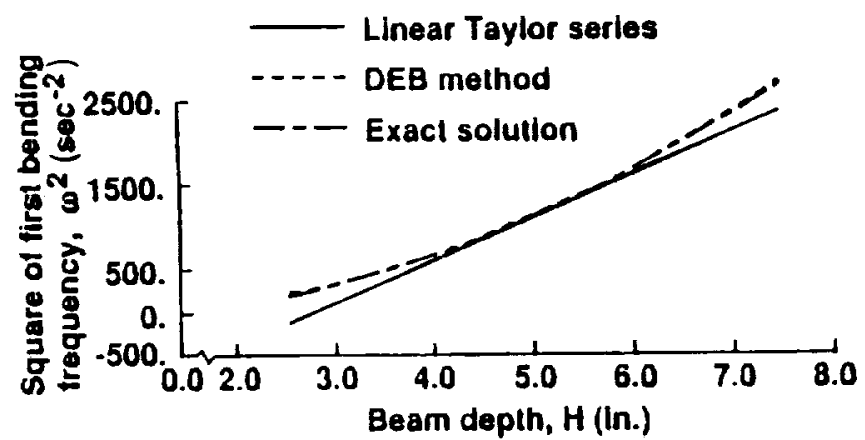

Fig. 2 Comparison of DEB and Taylor series approximations of frequency for perturbation of beam depth, $H(B=3.75$ in., $M=0.1 \mathrm{bm}$. ).

comparison between the approximations for the frequency due to a change in the magnitude of the tip mass. Again the new approximation is very accurate.
Only a four percent deviation from the exact solution is seen for a 100 percent increase in the tip mass compared to a ten percent deviation for the linear Taylor series approximation. Figure 4 illustrates the application

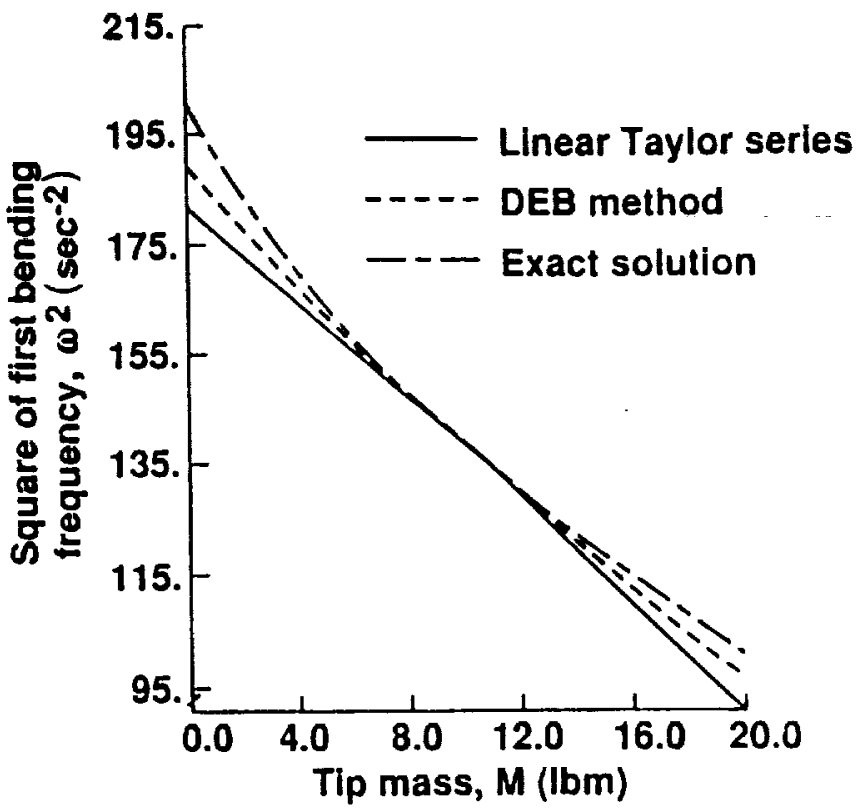

Fig. 3 Comparison of DEB and Taylor series approximations of frequency for perturbation of tip mass ( $B=3.75$ in., $H=5.0$ in.).

of the DEB method to simultaneous changes in three design variables: tip mass, bending inertia, and crosssectional area of the beam. Each step, $s$ represents a ten percent perturbation in the design variables from their nominal values. In comparing the DEB approximation with the exact solution there was a 36 percent error for 50 percent increase in the design variables. The linear Taylor series approximation was in error by nearly 100 percent. Figure 5 shows the DEB and linear Taylor series approximations of the second bending frequency compared to the exact solution for perturted values of the beam depth, $H$. Again, the new method is very accurate. For as much as a fifty percent increase in $\mathrm{H}$, the DEB approximation is within 2 percent of the exact solution compared to 12 percent for the linear Taylor series. This result is very similar to the result for the first bending mode with $\mathrm{H}$ as the design variable. Similar trends occurred in perturbation studies of the other variables for approximating frequencies of the first and second bending modes. 


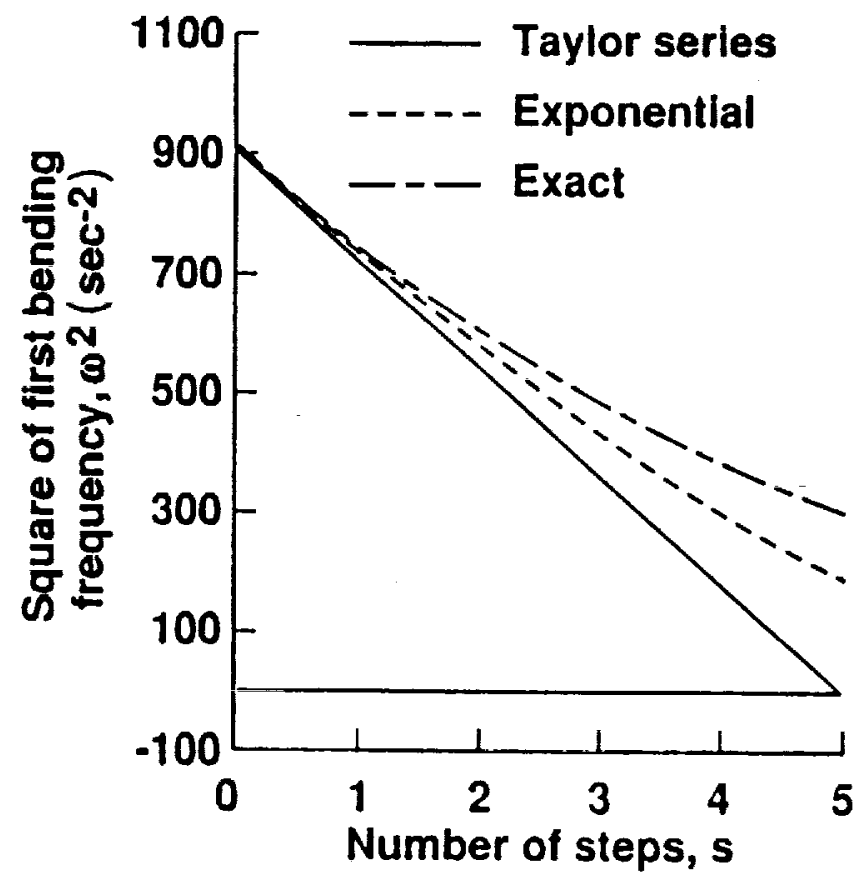

Fig. 4 Comparison of DEB and Taylor series approximations of frequency for simultaneous perturbation of tip mass $M$, bending inertia I, and crosssectional area $A$ ( Nominal values: $M=5.0 \mathrm{lbm}$., $\mid=28.863$ $\mathrm{in}^{4}, A=7.0 \mathrm{in}^{2}$ ).

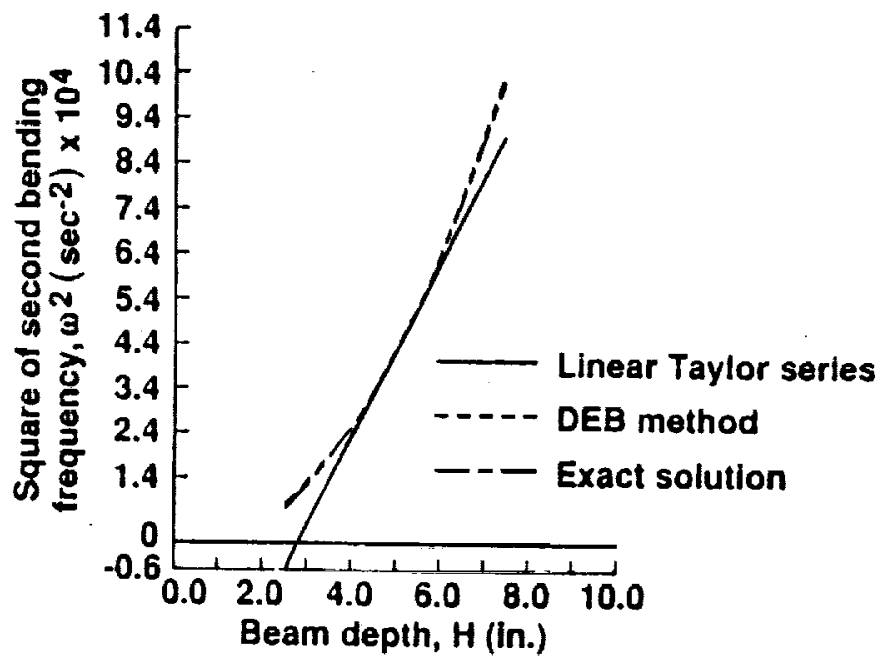

Fig. 5 Comparison of DEB and Taylor series approximations of frequency for perturbation of beam depth, $H(B=3.75$ in., $M=0$. Ibm. $)$.

\section{Mode Shape Aporoximation Results}

Figure 6 shows the first bending mode shape for the nominal design, and the linear Taylor series approximation, the DEB approximation, and the exact solution for a 50 percent increase in the beam width, $B$.
Figure 7 shows the second bending mode shape for the nominal design and both approximations and the exact shape for an 80 percent increase in the tip mass. Although the linear Taylor series approximation is very accurate, the DEB approximation is slightly closer to the exact curve in both figures. However, the mode shapes do not seem to be sensitive to design changes for this test problem as indicated in figures 6 and 7 .

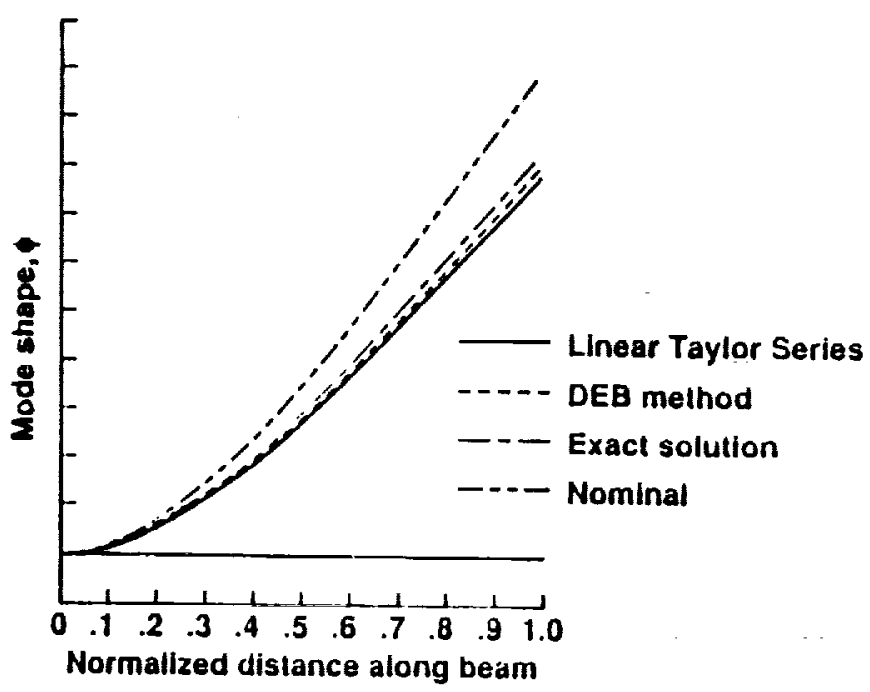

Fig. 6 Comparison of DEB and Taybr series approximations of first bending mode shape for 50 percent increase in beam width, $B$ (Nominal values: $H=5.0$ in., $B=3.75$ in. ).

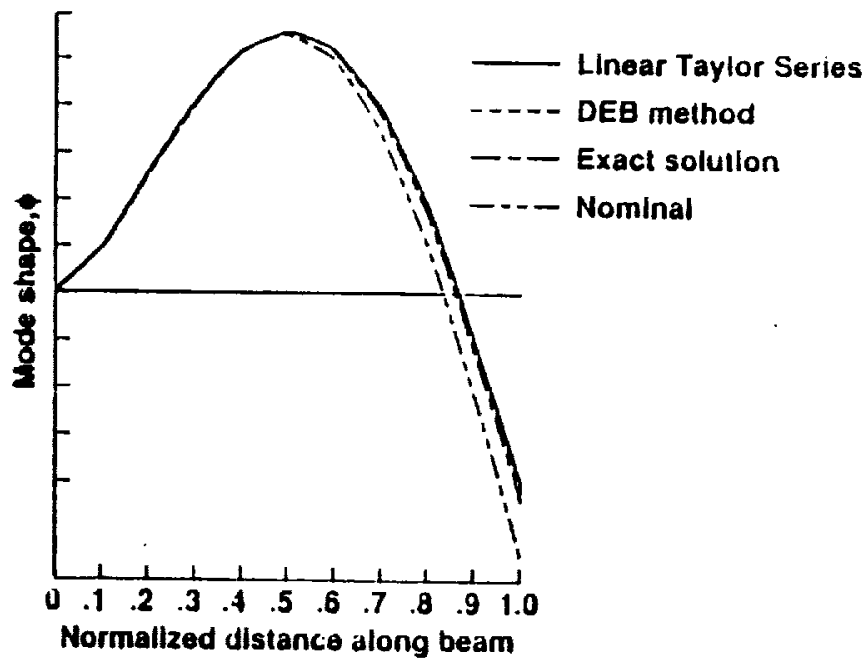

Fig. 7 Comparison of DEB and Taylor series approximations of second bending mode shape for 80 percent increase of tip mass, $M$ ( Nominal values: $B=3.75$ in., $H=5.0$ in., $M=5.0 \mathrm{lbm}$. ). 
In some cases the DEB method did not approximate the mode shapes as accurately as the linear Taylor series approach. Usually, these were cases in which the change in the mode shape due to perturbations in the design were small. Other test cases may provide a more challenging problem for testing these approximation methods for mode shapes and will be a topic for future investigation.

\section{Static Disolacement Approximation Results}

The cantilever beam of figure 1 was also used to test the method for approximating static displacements. The design variables were the dimensions $H$ and 8 . A cubically distributed loading was used to represent the applied force and the load values at each grid point of the model are given in table 2.

Table 2 Values of applied force at the grid points of the beam model

\begin{tabular}{|c|c|c|c|}
\hline Grid point & Force (bf) & Grid point & Force (lbf) \\
\hline 1 & 0.000 & 7 & 5.400 \\
\hline 2 & 0.025 & 8 & 8.575 \\
\hline 3 & 0.200 & 9 & 12.800 \\
\hline 4 & 0.675 & 10 & 18.225 \\
\hline 5 & 1.600 & 11 & 25.000 \\
\hline 6 & 3.125 & & \\
\hline
\end{tabular}

Figure 8 shows a typical convergence plot for the DEB method equation (16) for the maximum displacement. This figure suggests that the number of terms needed for a converged value is 3 or 4 . For this same number of terms and the same amount of computation, the Taylor series approximation (equation 19) is known to be more accurate and would be chosen over the DEB method unless it did not converge for certain values of $\Delta v$. Results for the linear Taylor series will be included because it is a frequently used method of approximation.

Figures 9, 10, and 11 show results for perturbation of the nonlinear design variable, $\mathrm{H}$. In figure 9, the maximum displacement for values of $\mathrm{H}$ from 3.5 inches to 8.5 inches was plotted for the DEB, linear Taylor series approximations, converged Taylor series and the exact solutions. As $\mathrm{H}$ is decreased, the bending stiffness of the beam is decreased causing the beam displacement to become very large. At this end of the range it is difficult for the approximation methods 10 follow the curvature of the exact results. At the other end, however, the DEB method appears to follow the trend of the exact curve. The linear Taylor series approximation gives negative values for the displacement beyond 40 percent perturbation of $\mathrm{H}$ from the nominal value.

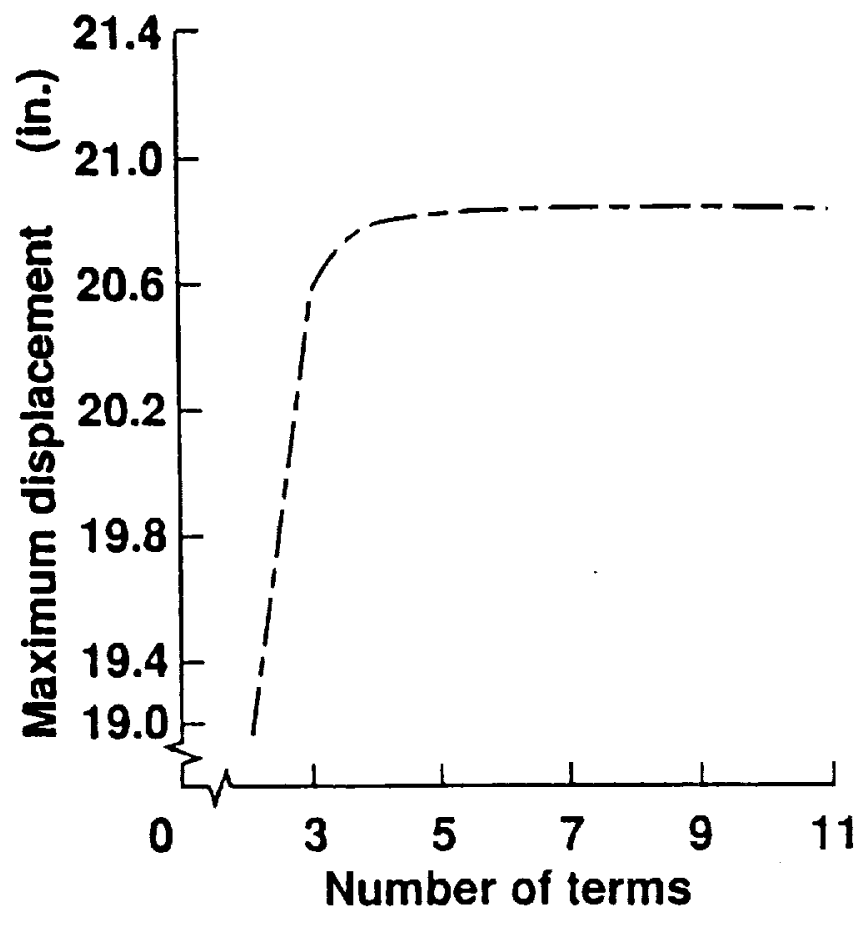

Fig. 8 Convergence plot of DEB method

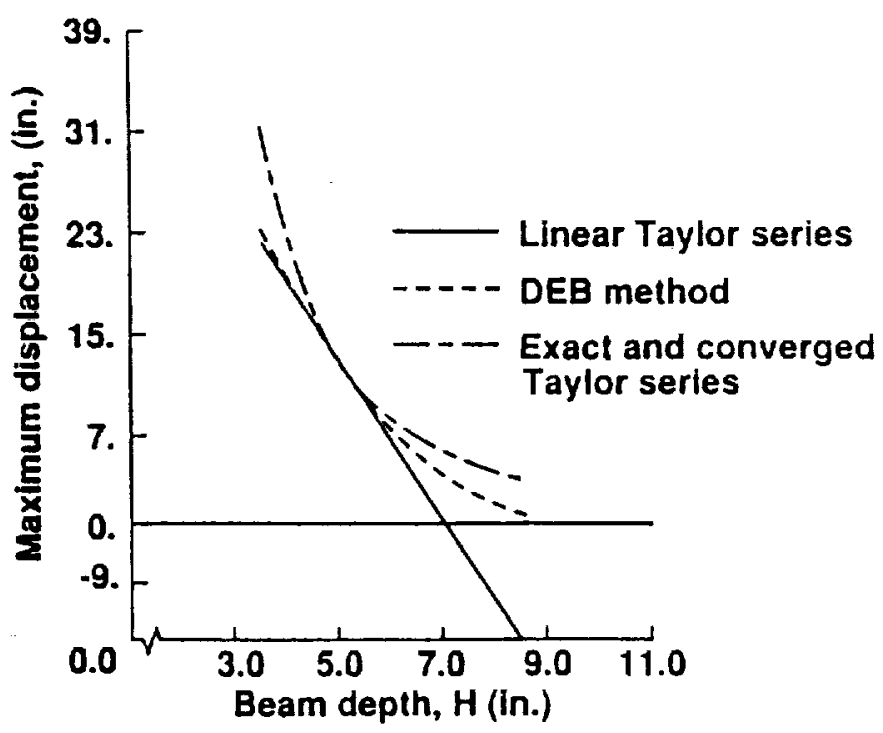

Fig. 9 Comparison of DEB and Taylor series approximations of maximum displacement versus perturbation of beam depth, $H$. 
Figure 10 is a graph of the displacement of the beam for a 30 percent decrease in the nonlinear variable $H$. The figure compares the DEB and linear Taylor series approximations to the exact solution. Both approximations are very good with the DEB method slightly more accurate.

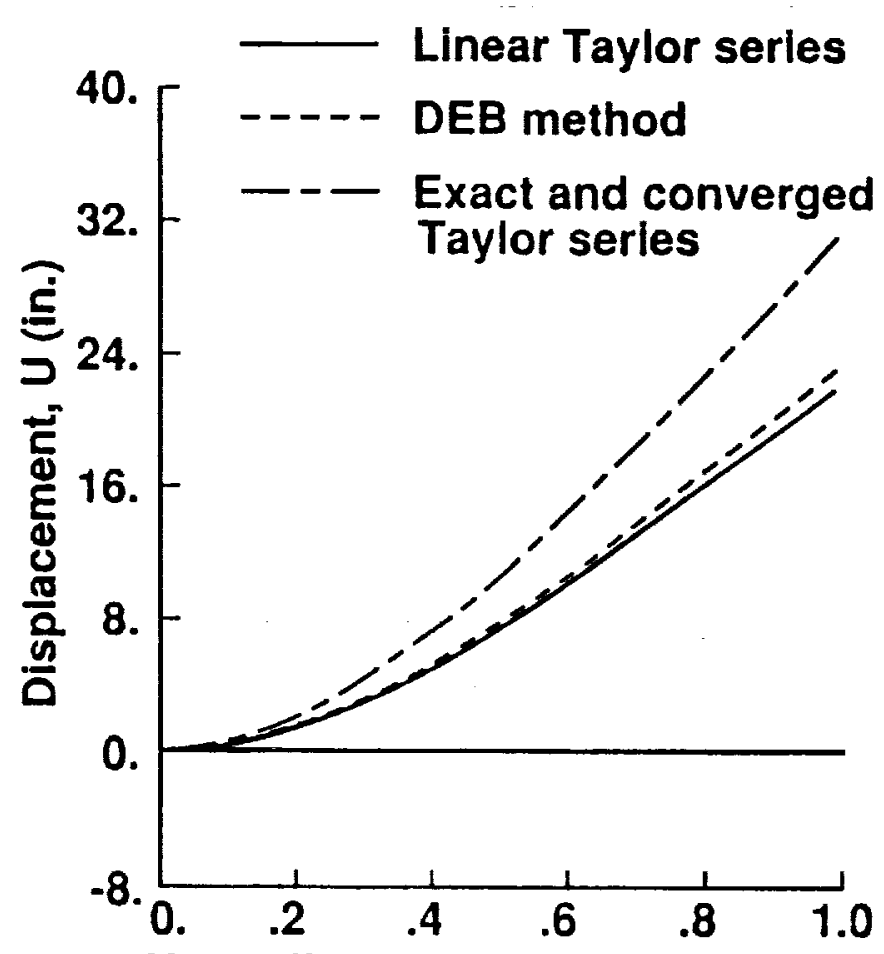

Normalized distance along beam

Fig. 10 Comparison of DEB and Taylor series approximations of displacement for 30 percent decrease in beam depth, $\mathrm{H}$.

A similar comparison is shown in figure 11. For a 50 percent increase in $\mathrm{H}$ from the nominal value, the DEB method had an error of 47 percent for the maximum displacement. However, the linear Taylor series approximation gives a displacement in the opposite direction. This is in accordance with the result we saw in figure 9.

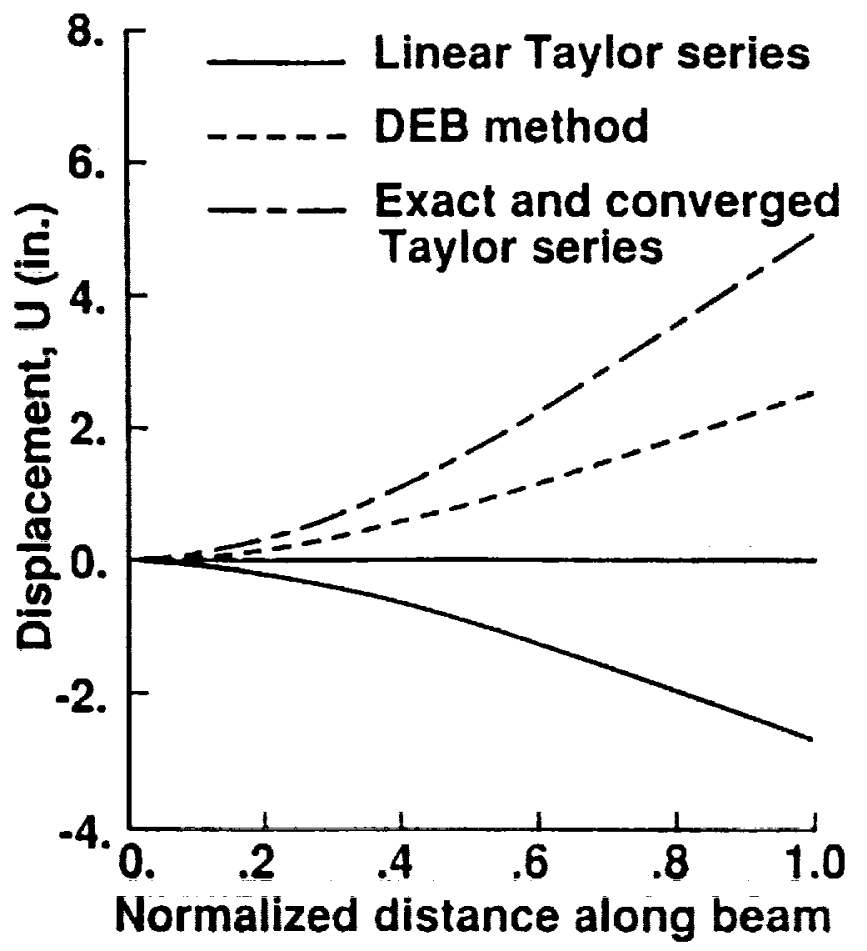

Fig. 11 Comparison of DEB and Taylor series approximations of displacement for 50 percent increase in beam depth, $H$.

Figures 12, 13, and 14 show results for linear Taylor series and DEB displacement approximations, converged Taylor series and exact solutions for the linear variable $B$. In figure 12, the maximum displacement for values of B from 1.875 inches to 5.625 inches was plotted for each approximation method and compared to the exact solution. Again, it appears to be more difficult to approximate the displacement for small beam dimensions, but the DEB method follows the exact curve, particularly for the larger values of $B$. Figure 13 shows a plot of the approximate displacements and the exact solution for a 50 percent decrease in $\mathrm{B}$. Both approximations are good but the DEB curve is closer to the exact. Figure 14 shows the result for a 50 percent increase in the design variable. It shows that the DEB approximation is within 8 percent of the exact solution compared to the linear Taylor series error of 21 percent. 


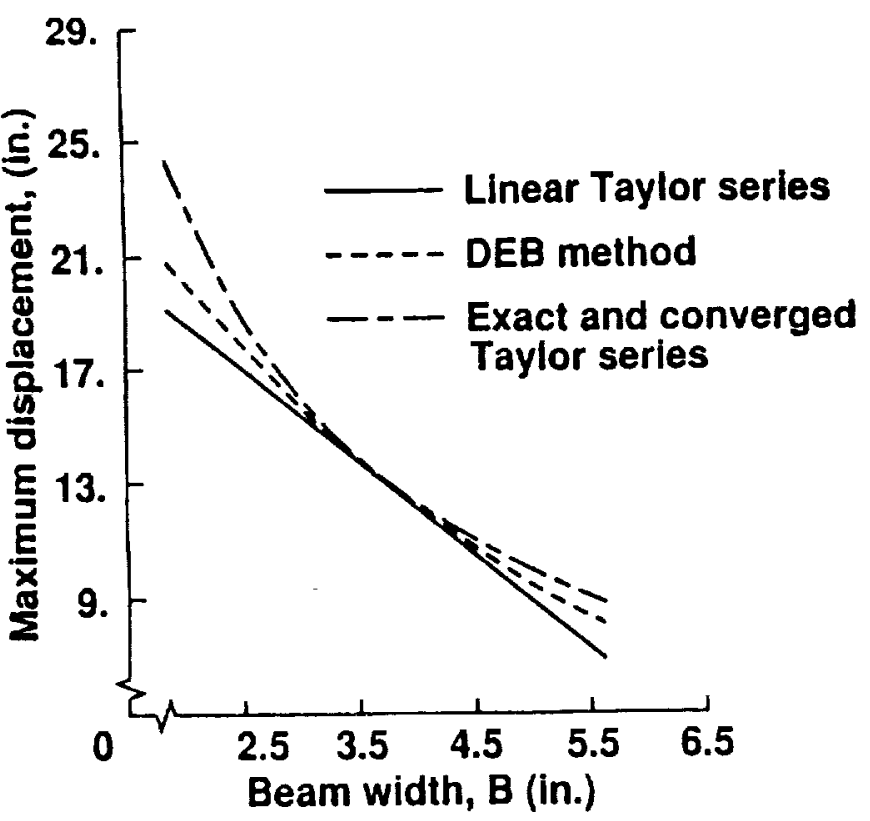

Fig.12 Comparison of DEB and Taylor series approximations of maximum displacement versus perturbation of beam width, $B$.

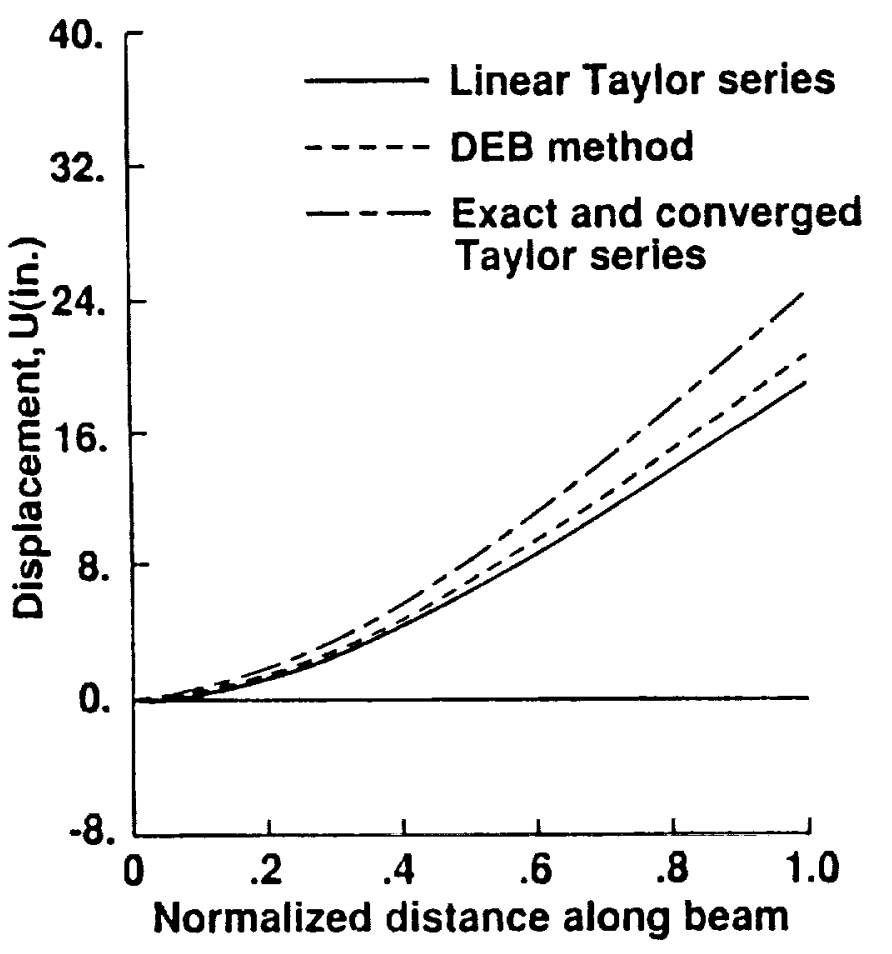

Fig. 13 Comparison of DEB and Taylor series approximations of displacement for 50 percent decrease in beam width, $B$.

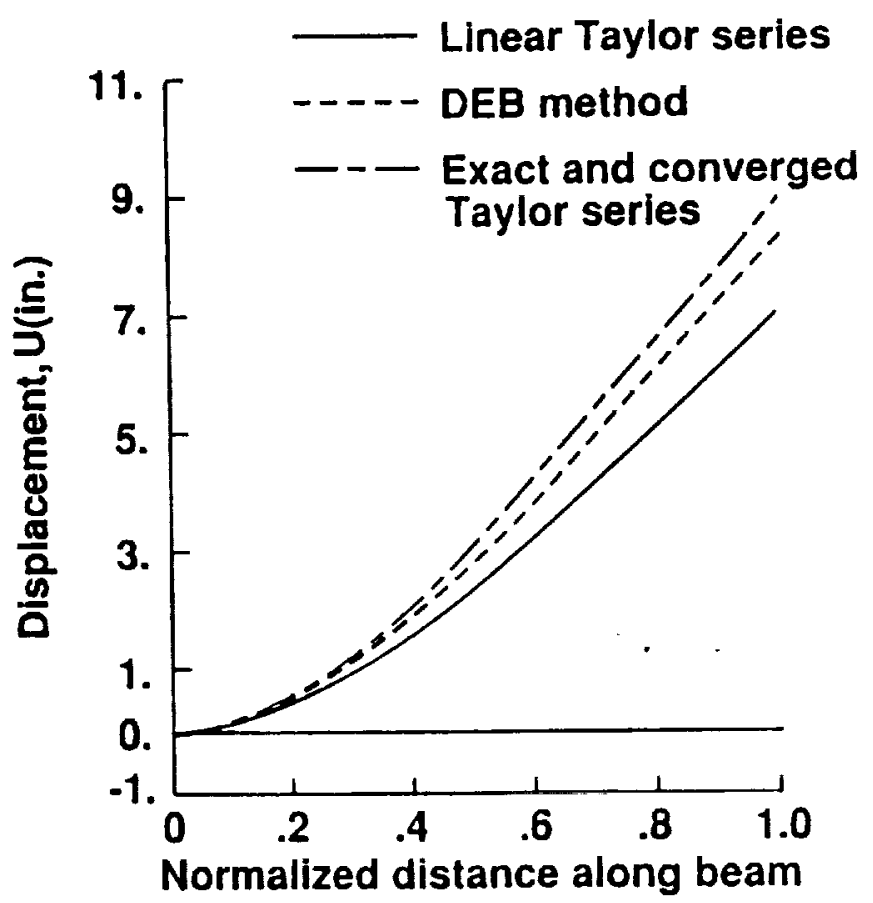

Fig. 14 Comparison of DEB and Taylor series approximations of displacement for 50 percent increase in beam width, $B$.

\section{Additional Aporoximations}

An additional application of the DEB approximation is in estimating system response quantities that depend on approximated quantities. Inserting the approximate displacement field into the stress-displacement equations to obtain approximate stress field is an example of this. Figure 15 shows a graph of maximum normal bending stress for perturbed values of $B$. The stresses were obtained using the approximate values of $U$ from the DEB method and the linear Taylor series approach and are compared to the converged Taylor series and exact solutions. The approximate stress field shown in figure 15 is very accurate. This was a particularly good result in view of the fact the the stresses are in effect proportional to the second derivatives of the approximate displacements. 


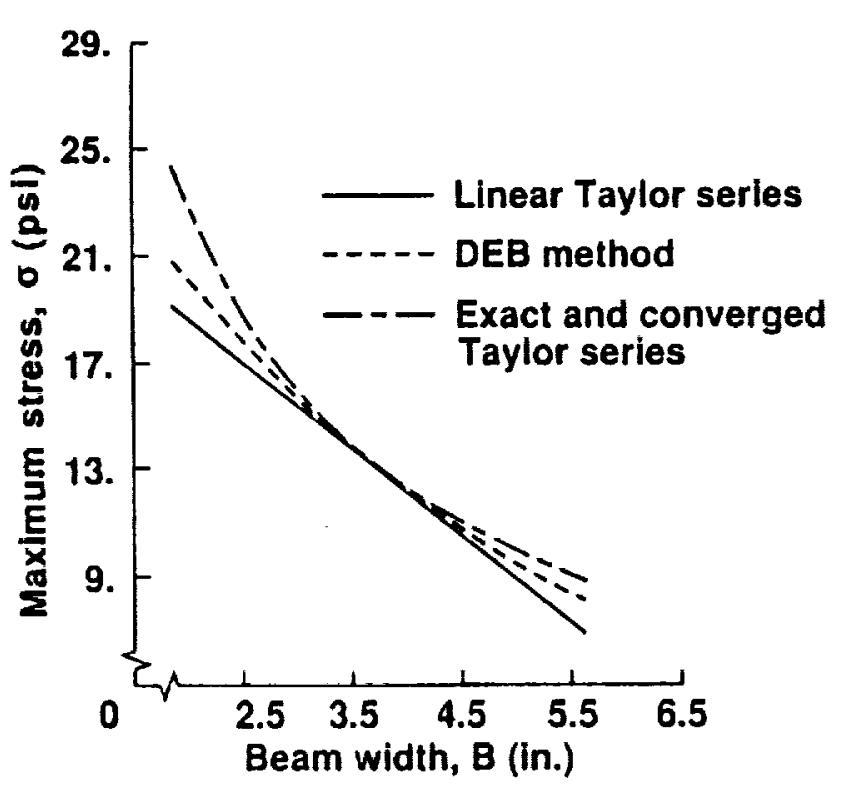

Fig. 15 Comparison of DEB and Taylor series approximations of maximum stress versus perturbation of beam width, $B$.

\section{Validity of Assumptions Made in DEB Aporoximation Method}

Recall that the methods developed in this paper were based on the assumption that certain variables were assumed constant, for example $a$ and $b$ in equations (5) and (26). In order to assess the validity of these assumptions, the actual values of the coefficients $a_{\theta}$ and $b_{\theta}$ were plotted for values of the tip mass, bending inertia, and cross-sectional area perturbed

simultaneously. Figure 16 shows a graph of $a_{\theta}$ and $\frac{b_{\theta}}{a_{\theta}}$ versus the number of 10 percent step increases in the design variables. The lower curve, shows that $\frac{b_{\theta}}{a_{\theta}}$ is essentially constant. The upper curve representing the coefficient $a_{g}$ shows a deviation of up to 19 percent from the nomial value when the design variables are changed by up to 50 percent. Assuming $a_{\theta}$ to be constant was not detrimental to the approximation results. As shown in figure 4, the DEB method was able to obtain very good approximations for the full range of the pertubed design variables.

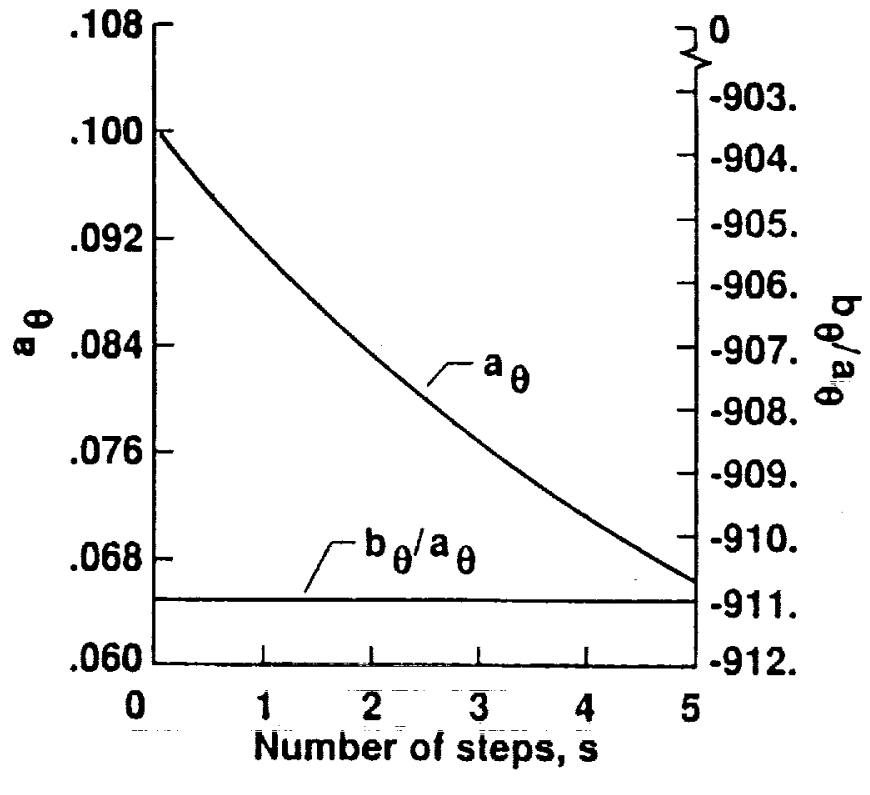

Fig. 16 Actual values of coefficients assumed constant in DEB frequency approximation for simultaneous perturbation of tip mass, bending inertia, and crosssectional area

Figure 17 shows a graph of the coefficients that appear in the mode shape approximation of equation (10). The plot of $D$ versus perturbed values of tip mass show plus and minus 20 percent deviations in $D$ based on up to 100 percent perturbation in the tip mass. The second plot shows the vector $\frac{Q}{D}$ for the nominal tip mass and for plus and minus 100 percent perturbations from the nominal mass. These plots show that the vector varied considerably, however, as shown in figure 6 , this did not seem to have a large effect on the approximate mode shapes obtained with the DEB method. 


\section{Beferences}
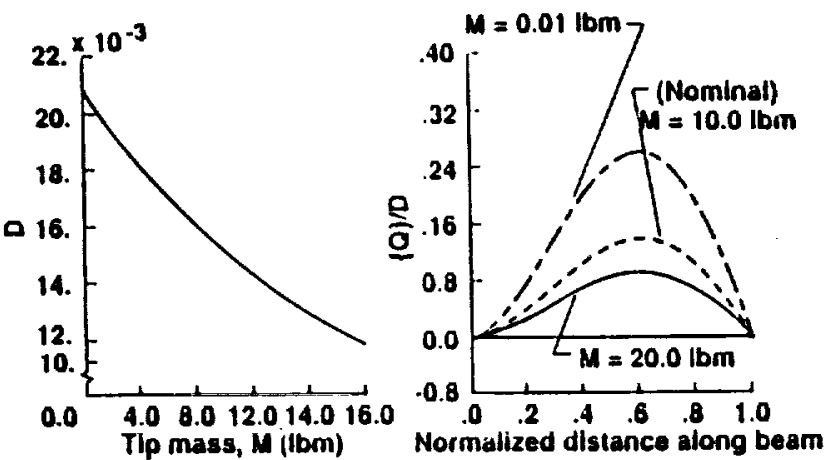

Fig. 17 Actual values of coefficients assumed constant in DEB mode shape approximation for mass perturbation

\section{Concludino Remarks}

This paper described a method that is based on interpreting sensitivity equations of structural response as differential equations that may be solved for closed form approximations. The method was developed for approximating vibration frequencies, mode shapes and static displacements. The test cases used to evaluate the approximations involved perturbations of the height, width, cross-sectional area, tip mass, and bending inertia of a cantilever box beam. The results were compared to the linear Taylor series approximations and exact solutions. The results showed that the new method is very accurate and in most cases more accurate than the linear Taylor series approximations. The method is applicable to both linear and nonlinear design variables and to simultaneous perturbation of several design variables. Also, the approximated response may be used to approximate behavior derived from the response, for example, approximating stresses from an approximate displacement field. To date, the method has been developed and demonstrated for frequencies, mode shapes, static displacements, and stresses derived from the displacement. In principle, the method is applicable to approximating any quantity for which an analytical sensitivity formula is available.

\section{Acknowledgement}

The authors wish to express their special thanks to Professor Raphael T. Haftka of Virginia Polytechnic Institute and State University for his valuable discussions and suggestions during the course of this research.
${ }^{1}$ Walsh, J. L., "Application of Mathemetical Optimization Procedures to a Structural Model of a Large FiniteElement Wing," NASA TM-87597, January 1986.

2 Storaasli, 0 . 0 . and Sobieszczanski, J., "On theAccuracy of the Taylor Approximation for Structure Resizing," AIAA Journal, Vol. 12, 1974, pp. 231-233.

${ }^{3}$ Haftka, R. T. and Shore, C. P., "Approximate Methods for Combined Thermal Structural Design," NASA TP. 1428, 1979.

4 Haftka, R. T., "Design for Temperature and Thermal Buckling Constraints Employing a Noneigenvalue Formulation," Journal of Spacecratt, Vol. 20, 1983, pp. 363-367.

5 Salajegheh, E. and Vanderplaats, G. N., "An Efficient Approximation Method for Structural Synthesis with Reference to Space Structures," Space Structures Journal, Vol. 2, 1986/1987, pp. 165-175.

6 Vanderplaats, G. N. and Salajegheh, E., "New Approximation Method for Stress Constraints in Siructural Synthesis," AIAA Journal, Vol. 27, No. 3 , March, 1989.

7Schmit, L. A. and Farshi, B., "Some Approximation Concepts for Structural Synthesis," AlAA Journal, Vol. 12, No. 5, 1974, pp.692-699.

8 Lust, R. V. and Schmit, L. A., "Alternative Approximation Concepts for Space Frame Synthesis," AIAA Journal, Vol. 24, October 1986. pp.1676-1684.

${ }^{9}$ Starnes, J. H., Jr. and Haftka, R. T., "Preliminary Design of Composite Wings for Buckling Stress and Displacement Constraints," Journal of Aircraft, Vol. 16 , 1979, pp.564.570.

10prasad, B., "Potential Forms of Explicit Constraint Approximations in Structural Optimization-Part I:Analysis and Projections," Computer Methods for Applied Mechanical Engineering, 1983, pp. 245-261.

11 Inamura, T., "Eigenvalue Reanalysis by Improved Perturbation," International Journal for Numerical Methods in Engineering, Vol. 26, 1988, pp. 167-181.

12Fox, R. L. and Kapoor, M. P., "Rate of Change of Eigenvalues and Eigenvectors," AIAA Journal, Vol. 6 , December 1968, pp. 2426-2429.

13 Nelson, R. B., "Simplified Calculation of Eigenvector Derivatives," AIAA Journal, Vol. 14, No. 10, 1976, pp. 1201-1205.

14 Kirsch, U., Optimum Structural Design, McGraw Hill, New York, 1981 , pp.67.

15Whetstone, W. D., "Engineering Analysis Language Reference Manual-EISI-EAL System Level, 2091," Engineering Information Systems, Inc. EISI-EAL, July 1983. 


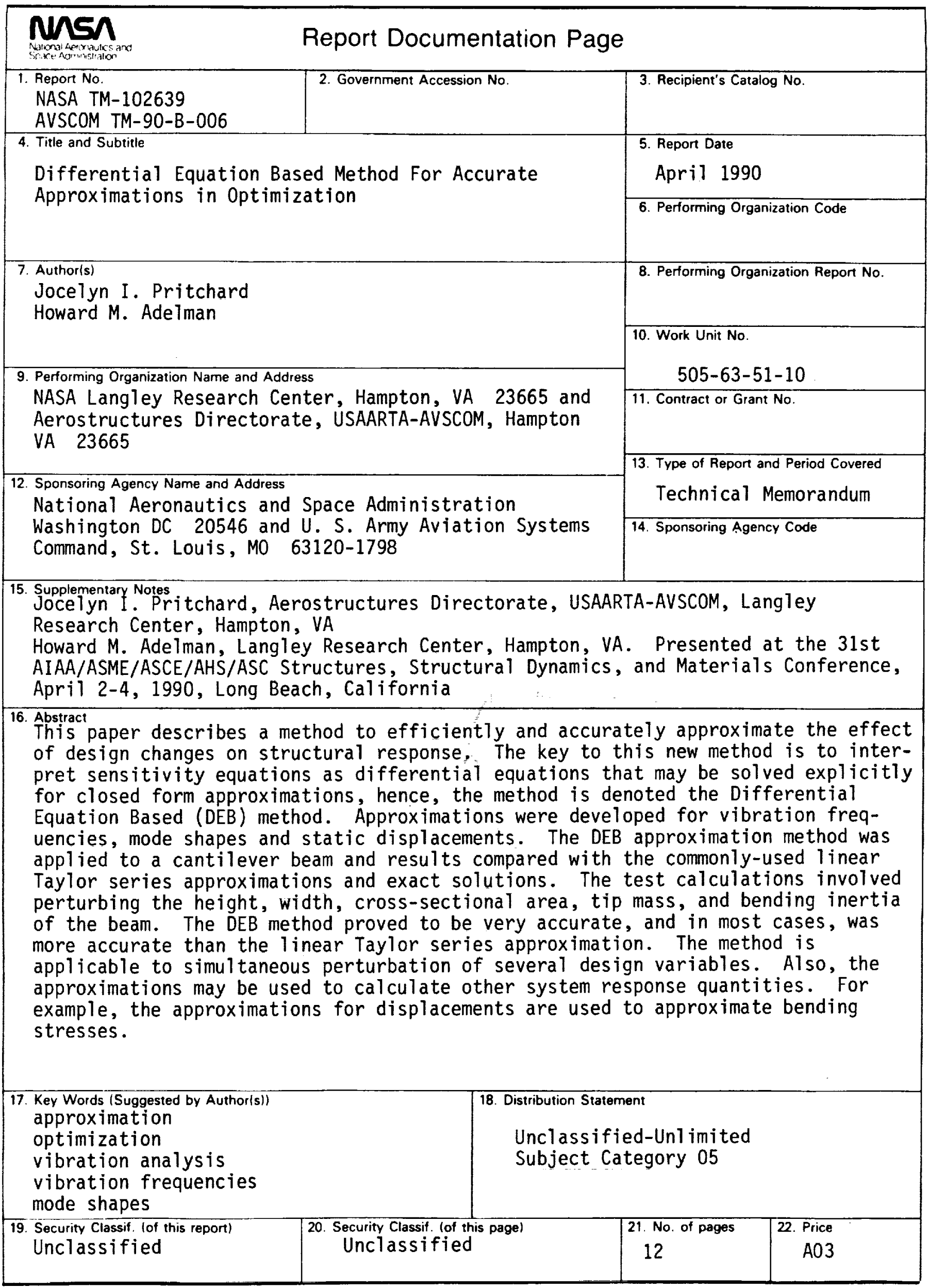

